\title{
Use of steel substrates in diffractive optics: Near field of high surface quality steel tape gratings
}

\author{
Francisco Javier Salgado-Remacha*, Francisco Jose Torcal-Milla, Luis Miguel Sanchez-Brea, \\ Eusebio Bernabeu \\ Universidad Complutense de Madrid, Applied Optics Complutense Group, Optics Department, Facultad de Ciencias Físicas, Ciudad Universitaria s.n., 28040 Madrid, Spain
}

\section{A R T I C L E I N F O}

\section{Article history:}

Received 24 March 2010

Received in revised form

14 September 2010

Accepted 19 October 2010

Available online 12 November 2010

Keywords:

Diffraction gratings

Talbot effect

Rough surfaces

Steel tape

\begin{abstract}
A B S T R A C T
Steel tape is used in optical applications due to its mechanical properties. However, roughness of steel surfaces affects to wave propagation. The effect can be observed using diffraction gratings with low periods. In such a case, self-images of the grating appear at certain periodic distances from the grating (Talbot planes). When standard steel is used, the contrast of Talbot self-images strongly decreases with the distance from the grating. In this work, we prove that controlling the surface quality of steel, it is possible to improve its optical behavior and, as a consequence, high quality surface steel represents a good choice for diffractive optics when the use of chrome-on-glass masks is not indicated. As an example, we have manufactured a diffraction grating over a high quality steel surface by means of an ablation process with a nanosecond pulsed laser. The contrast of the self-images for these gratings decreases very slowly with the distance, in comparison with the self-images obtained with standard steel tape gratings.
\end{abstract}

(c) 2010 Elsevier Ltd. All rights reserved.

\section{Introduction}

Diffraction gratings are one of the most used optical elements, since they can be applied in a high number of different branches of science (chemistry, photonics, astrophysics, engineering, biology, etc.), and also in several applications, such as telescopes, machinetool, spectroscopy, precision optical metrology, etc. [1]. Chrome on glass gratings are commonly used for precision optical metrology purposes [2]. These gratings are formed by chrome strips on a glass substrate and their optical behavior is near to the ideal. However, there exist applications that require using gratings with a long length (more than $3 \mathrm{~m}$ ). In such a case, glass gratings are not appropriate since they result hard to manufacture and handle. Therefore, steel tape gratings are preferred [3]. Unfortunately, they present a worse optical behavior due to surface roughness. This roughness affects to the optical properties of the gratings. For example, the effect over Talbot self-images is notorious and it has been analyzed in previous works [4,5]. Talbot effect consists of the replication of the intensity pattern at some certain distances from the grating when it is illuminated by a collimated beam [6]. When the grating is engraved over a rough surface, the contrast of Talbot self-images decreases with the distance between the grating and the observation plane. Therefore, the behavior of the devices that use steel tape gratings gets worse. This decreasing depends strongly on the roughness parameters.

\footnotetext{
* Corresponding author.

E-mail address: fjsalgado@fis.ucm.es (F.J. Salgado-Remacha).
}

In this work, we show the conditions in which steel tapes can be used in diffractive optics applications. In particular we have focused on the self-imaging process. We experimentally investigate the effect of the roughness parameters on the contrast of the self-images. Steel tape gratings have been manufactured using a laser ablation station over two steel substrates with different levels of roughness. Talbot self-images contrast was obtained for each grating. We show that for higher surface quality, the contrast of the self-images does not decrease significantly for standard working distances (around millimeters). The experimental results are compared with the theoretical model showing that controlling the surface quality of the steel tape it is possible to achieve a highquality grating, acting close to the ideal behavior of a grating (like a chrome on glass grating).

\section{Theoretical fundamentals}

In previous works, the authors developed a formalism to describe the behavior of steel tape gratings in the near field. One of the effects of roughness is the decreasing of the contrast of the self-images with the distance grating-observation plane $[4,5]$ The self-images appear at distances given by $z_{T}=m p^{2} / \lambda$, where $p$ is the period of the grating, $\lambda$ the illumination wavelength and $m$ entire numbers. Since the roughness follows a random distribution, it must be described in terms of statistical parameters such as the correlation length, $T$, and the standard deviation of heights, $\sigma$. A Gaussian distribution on heights and a decreasing exponential 
autocorrelation function are assumed to describe the roughness over the surface $[7,8]$. We have selected this functions based on an experimental analysis, as it is shown in Section 3.1.

Considering monochromatic plane wave, the field can be calculated by solving

$U\left(x_{2}, z\right)=A_{0} \sum_{n} a_{n} \int_{-\infty}^{\infty} \exp ($ iqxn $) \exp \left[\frac{i k}{2 z}\left(x_{2}-x\right)^{2}\right] r(x) d x$,

where $r(x)$ is the reflectivity of the surface and it is obviously unknown due to the random nature of the surface. Therefore, it is not possible to analytically determine the field after the grating. Although, it is possible to obtain the mean intensity as follows. Considering that the roughness has a Gaussian distribution in heights, a decreasing exponential correlation function and that higher order surface properties do not affect, let us take the characteristic function of the roughness given in [7, Section 5.3]

$\chi_{2}=\exp [-g(1-C)]$

where $g=(2 k \sigma)^{2}, k=2 \pi / \lambda$, and $C$ is the correlation function. Now, choosing as correlation function, [8, Section 2.1.2], a decreasing exponential function, $C=\exp (-|\tau| / T)$ with $\tau=x-x^{\prime}$, which can be approximated for high roughness limit to $C \simeq 1-|\tau| / T$, [7]. Substituting this expression in Eq. (2) we obtain the characteristic function of the roughness for high roughness limit, $\left\langle r(x) r^{*}\left(x^{\prime}\right)\right\rangle=\exp \left(-\left|x-x^{\prime}\right| / T_{0}\right)$, being $T_{0}$ the optical correlation length of the field after the grating [7]. This parameter for a decreasing exponential function is related to the roughness by means of $T_{0}=T /(2 k \sigma)^{2}$, [9]. The statistical parameters, $T$ and $\sigma$, corresponds to the surface before the engraving process. After the ablation process, the surface topology changes in the zones where a

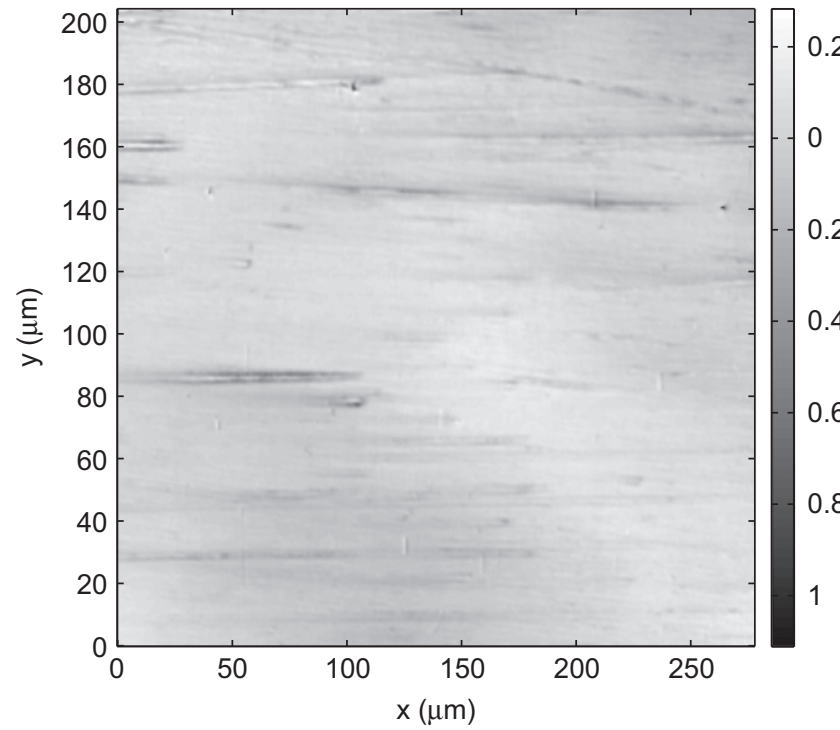

b

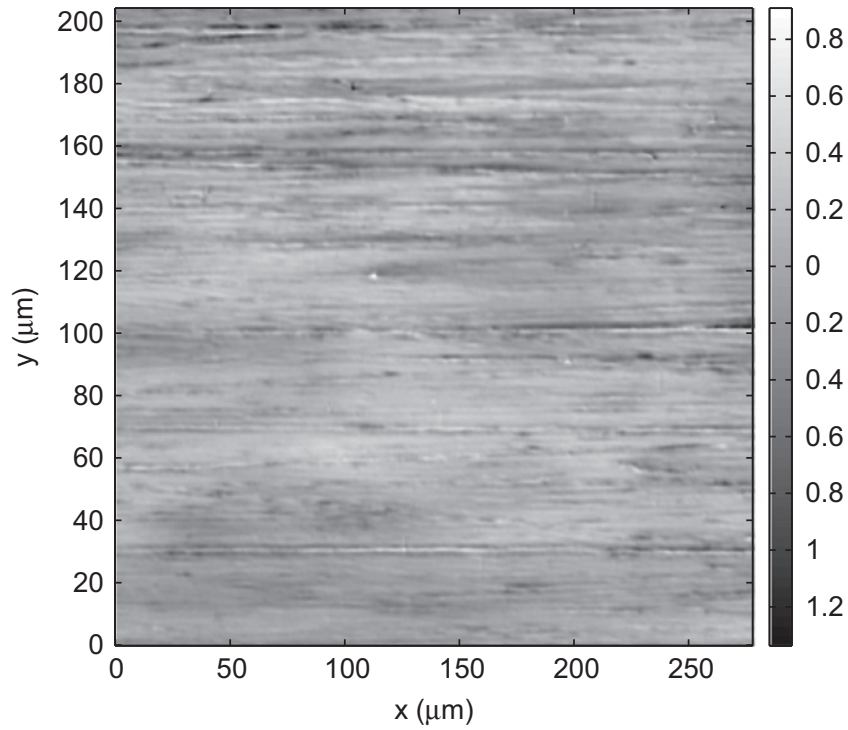

Fig. 1. Rough surfaces, obtained by means of confocal microscopy: (a) low roughness steel and (b) high roughness steel, height in microns.

a

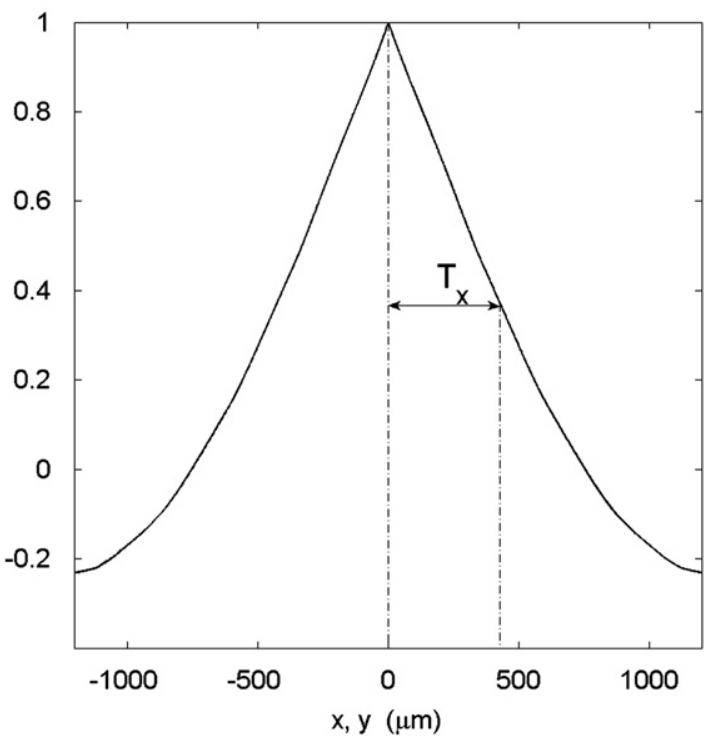

b

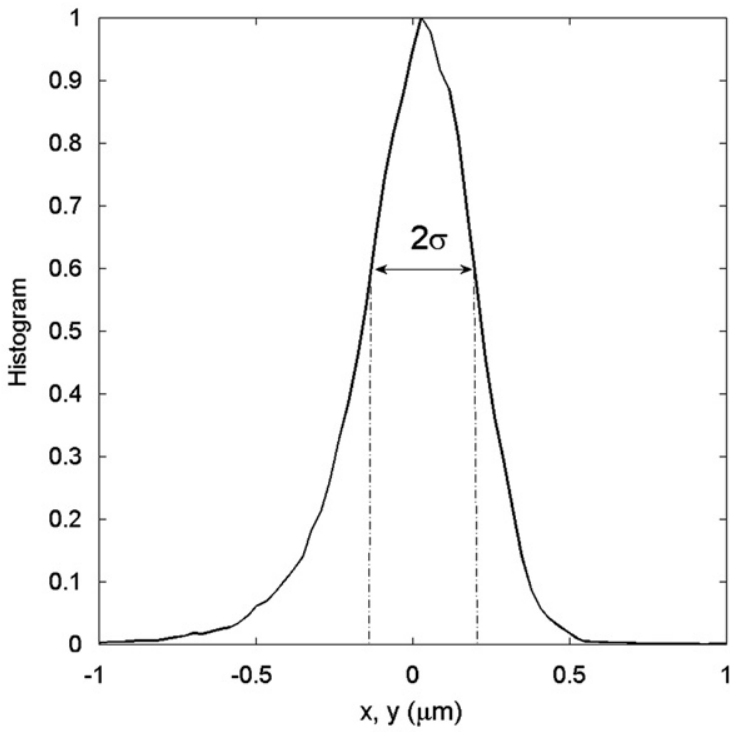

Fig. 2. Example of topographical measurements of steel tape surface: (a) correlation function of a rough surface and (b) heights histogram. 
ablation occurred. This new roughness is much higher than the original one and it acts like a scatterer, redirecting light into all directions, $[5,10]$ and contributing to the diffracted intensity like a background factor.

The mean intensity in the near field can be calculated solving [4]

$$
\begin{aligned}
\left\langle I\left(x_{2}, z\right)\right\rangle= & \left|A_{0}\right|^{2} \sum_{n, n^{\prime}} a_{n} a_{n^{\prime}}^{*} \int_{-\infty}^{\infty} \int_{-\infty}^{\infty} \exp \left[i q\left(x n-x^{\prime} n^{\prime}\right)\right] \\
& \times \exp \left\{\frac{i k}{2 z}\left[\left(x_{2}-x\right)^{2}-\left(x_{2}-x^{\prime}\right)^{2}\right]\right\} \exp \left(-\frac{\left|x-x^{\prime}\right|}{T_{0}}\right) d x d x^{\prime},
\end{aligned}
$$

where $A_{0}$ is the amplitude of the wave, $a_{n}, a_{n^{\prime}}^{*}$ are the Fourier coefficients of the grating with $n, n^{\prime}$ entire, $q=2 \pi / p$ and resulting in [4]

$$
\begin{aligned}
\left\langle I\left(x_{2}, z\right)\right\rangle= & \left|A_{0}\right|^{2} \sum_{n} \sum_{n^{\prime}} a_{n} a_{n^{\prime}}^{*} \exp \left[i q x_{2}\left(n^{\prime}-n\right)\right] \exp \left[-i \frac{q^{2}}{2 k}\left(n^{\prime 2}-n^{2}\right) z\right] \\
& \times \exp \left(-\frac{z}{w}\left|n-n^{\prime}\right|\right)
\end{aligned}
$$

with $w=p T_{0} / \lambda=p \lambda T /(4 \pi \sigma)^{2}$. The first exponential factor indicates the period of the self-images, the second exponential factor indicates the location of the self-images along the $z$-axis and the third exponential factor describes the decreasing of the global intensity along propagation axis owing to the surface roughness. The parameter $w$ describes the distance at which the intensity reduces a ratio $1 / e$. This fact is hugely detrimental since the gratings usually need to be separated from the detector for an appropriate design of the devices that use steel tape gratings.

The $90 \%$ of the total energy is with the orders $-1,0,1$. Then, we can truncate the summations in Eq. (4) up to first order. Then the

Table 1

Roughness data obtained with a confocal microscope, for the two steel samples.

\begin{tabular}{lllll}
\hline & $T_{x}$ & $T_{y}$ & $\sigma$ & $w_{x}$ \\
\hline High roughness substrate & $505.86 \mu \mathrm{m}$ & $44.23 \mu \mathrm{m}$ & $0.182 \mu \mathrm{m}$ & $2.92 \mathrm{~mm}$ \\
Low roughness substrate & $407.24 \mu \mathrm{m}$ & $118.71 \mu \mathrm{m}$ & $0.062 \mu \mathrm{m}$ & $22.20 \mathrm{~mm}$ \\
\hline
\end{tabular}

a

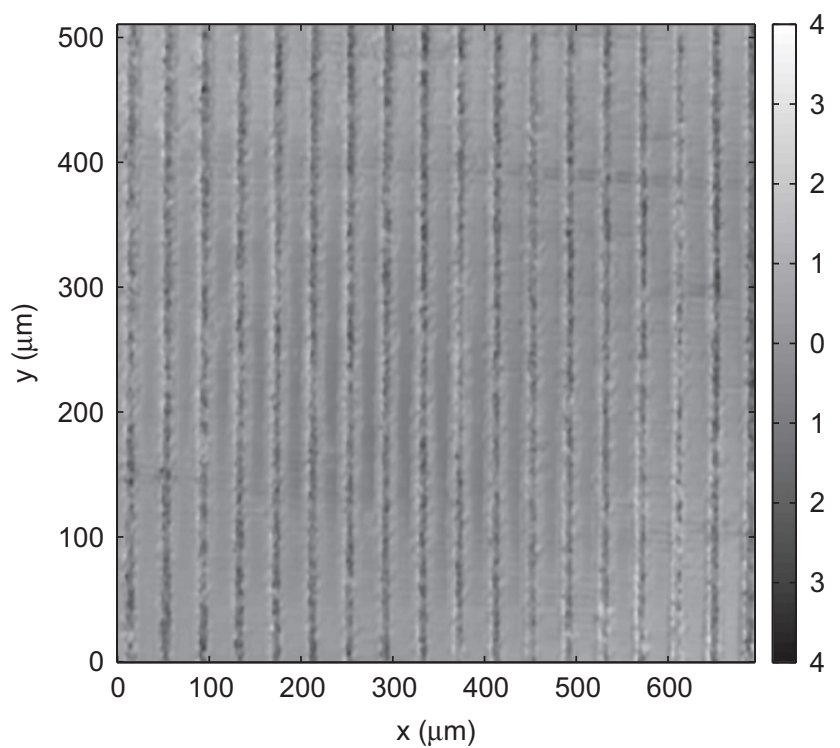

intensity simplifies to

$$
\begin{aligned}
\left\langle I\left(x_{2}, z\right)\right\rangle /\left|A_{0}\right|^{2} \approx & a_{0}{ }^{2}+2 a_{1}^{2}+4 a_{0} a_{1} \cos \left(q x_{2}\right) \cos \left[q^{2} z /(2 k)\right] \\
& \times \exp (-z / w)+2 a_{1}{ }^{2} \cos \left(2 q x_{2}\right) \exp [-z /(w / 2)],
\end{aligned}
$$

where we have assumed an amplitude binary grating $\left(a_{1}=a_{-1}\right.$ and the Fourier coefficients of the grating are real).

A way to measure the behavior of the grating is by measuring the contrast, which is usually defined as

$C(z)=\frac{I_{\max }(z)-I_{\min }(z)}{I_{\max }(z)+I_{\min }(z)}$,

where $I_{\max }(z)=\max (\langle I(x, z)\rangle)_{x} \quad$ and $\quad I_{\max }(z)=\max (\langle I(x, z)\rangle)_{x}$ are the maximum and minimum intensity for each observation plane, $\max ()_{x}$ and $\min ()_{x}$ stand for the maximum and the minimum along $x$-axis at a certain plane $z$, respectively. Therefore, the contrast depends on the distance $z$ from the grating, and this dependence is ruled by the roughness of the surface through $w$. The general functional equation to give the contrast involves eight infinite sums, but we can give an approximate expression from Eqs. (5) and (6) resulting in

$\langle C(z)\rangle=\frac{8 a_{0} a_{1} \exp (-z / w)}{2\left|a_{0}\right|^{2}+4\left|a_{1}\right|^{2}\{1+\exp [-z /(w / 2)]\}}$.

\section{Experimental approach}

According to Eqs. (4) and (7), the contrast of the self-images decreases slower when the surface roughness of the steel tape decreases. In order to verify this theoretical assumption, we have fabricated diffraction gratings over two different steel samples with different surface quality. We have obtained the value of $w$ from topological measurements. The resulting decreasing exponential is then compared with the experimental decaying of the Talbot self-images. Following we show the experimental approach used and the obtained results.

\subsection{Fabrication and characterization of steel tape gratings}

Topological images of both steel substrates, obtained using a confocal microscope ( $P l \mu$ by Sensofar, Barcelona, Spain), are shown

b

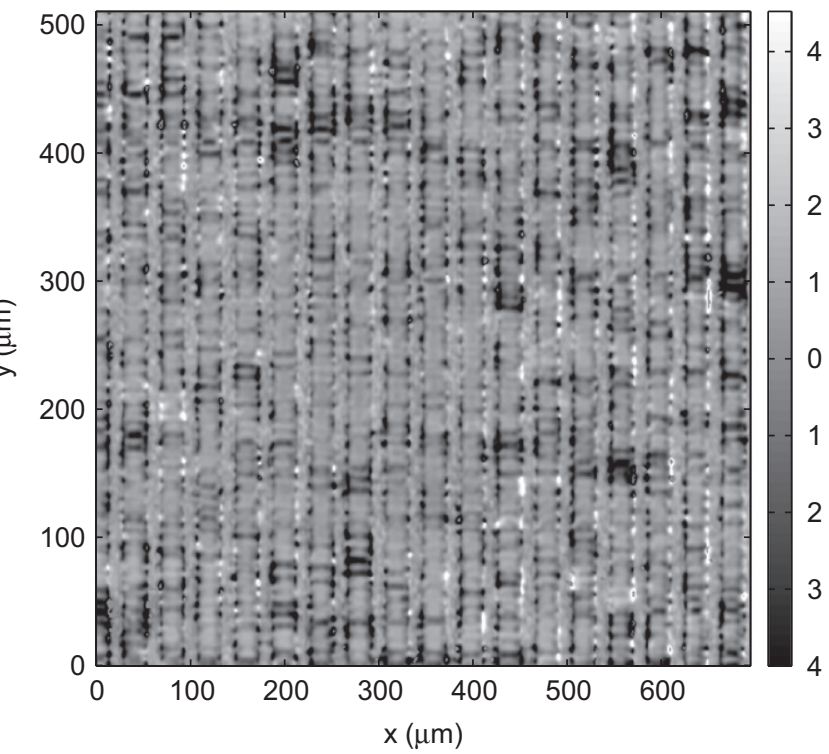

Fig. 3. Rough reflection grating, over a steel tape: (a) low roughness steel and (b) high roughness steel. 
in Fig. 1a and b. This kind of microscope is indicated for threedimensional measurements of surfaces since it allows us to calculate the roughness parameters from the surface topologies. We have computed the correlation lengths $T$ taking the semi-width of the autocorrelation peak at $1 / e$ of its height. Similarly, we obtain $\sigma$ from the heights histogram, as the semi-width at $1 / \sqrt{e}$. Examples of this measurements are shown in Fig. 2a (the correlation function) and in Fig. 2b (heights histogram). The shape of an exponential function for the correlation function and a Gaussian function for the heights histogram appears here clearly. For a more realistic measure, we have computed both parameters over an ensemble of 488 samples. The results are collected in Table 1, where we show the mean values over an ensemble of 488 linear measurements. Steel tapes present a strong anisotropy due to the manufacturing processes. For this reason, we present two different correlation lengths, one for each orthogonal direction $\left(T_{x}\right.$ and $\left.T_{y}\right)$. We perform an integration of the intensity along the axis parallel to the strips. We carry out this integration because we base on the operating system of an optical linear encoder and with this integration we can obtain an average in our measurements. Then, effects due to roughness along $y$-axis are avoided, and we only need $T_{x}$ for our calculations since it is the roughness that affects to the decreasing of the Talbot self-images contrast [10].

Once we have the roughness description for each surface, we calculate $w$ as

$w=w_{x}=\frac{p \lambda T_{x}}{(4 \pi \sigma)^{2}}$

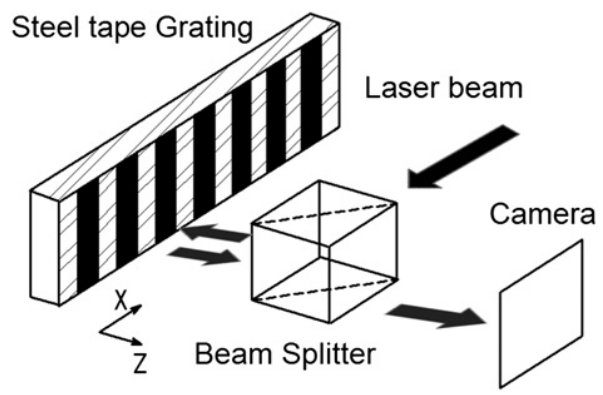

Fig. 4. Scheme of the experimental set-up.

a

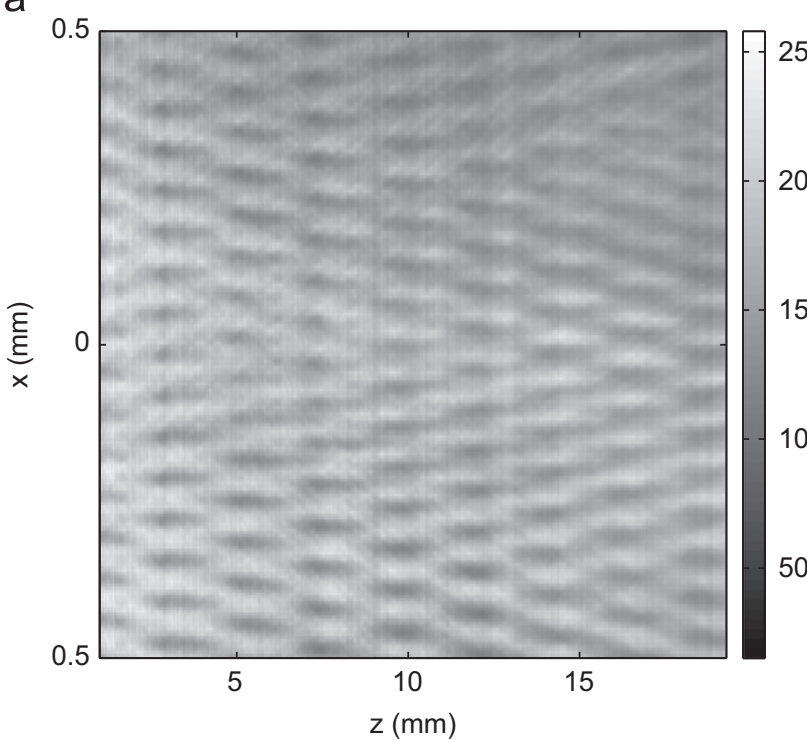

which appears in the last exponential term of Eq. (4). Then, we only need one parameter to know the optical behavior of the grating in the near field. We will make use of this parameter to compare our results. The values of $w$ are also shown in Table 1.

The gratings were engraved using a nanosecond pulsed laser ablation system, with a spot size about $5 \mu \mathrm{m}$. The effect of the ablation laser is to remove material and modify the surface topology, obtaining zones with different roughness. The parameters of the laser stage (that is, the intensity over the samples) were chosen in order to minimize the removal of material, modifying only the surface roughness. Then, the mean height remains the same. With this requirement, we ensure that the grating acts like an amplitude binary grating. Images of the diffraction gratings are shown in Fig. 3.

\subsection{Experimental measurement of self-images}

In order to corroborate our approach, we perform the experimental set-up shown in Fig. 4. A monochromatic collimated laser beam of wavelength $\lambda=675 \mathrm{~nm}$ (MC67070 model by Monocrom) impinges on a steel tape grating $(p=40 \mu \mathrm{m})$ with normal incidence with respect to its surface. The diffracted light crosses a beam splitter and is collected by a CCD camera (DFK 31BF03 model by Imagine Source, pixel size: $4.65 \times 4.65 \mu \mathrm{m}^{2}$ ) with a $10 \times$ microscope objective. The camera is placed on a motorized linear stage travelling along the $z$ direction, so it can collect images at any desirable distance.

As we have mentioned, we perform a vertical average (in the direction of the grating strips) for each plane, to obtain the evolution of the intensity diffracted by the grating (Fig. 5). Using these averaged images, we calculate the contrast for different distances $z$ between the grating and the observation plane. The contrast evolution along $z$-axis for both kinds of steel is shown in Fig. 6. The dependence on roughness clearly appears, in the form of a decreasing of the contrast. We plot two theoretical fittings to the experimental self-images, one using almost the complete sums and the other one using Eq. (7). To measure the contrast, we have used a variogram-based technique, since it provides an accurate measurement of contrast for experimental noisy measurements [11]. To validate the theoretical predictions, we have plotted also the exponential functions ruled by $F(z)=a \exp (-z / w)$, where $w$ was obtained from roughness data using Eq. (8) (this parameter is also

b

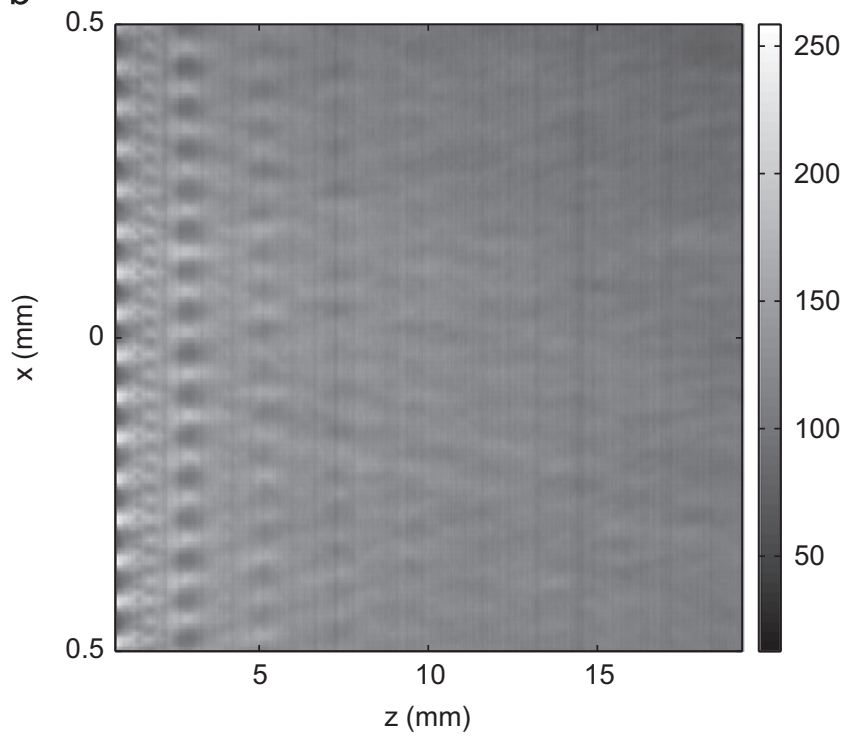

Fig. 5. Evolution of Talbot self-images along z-axis: (a) low level roughness steel and (b) high level roughness steel. 
a

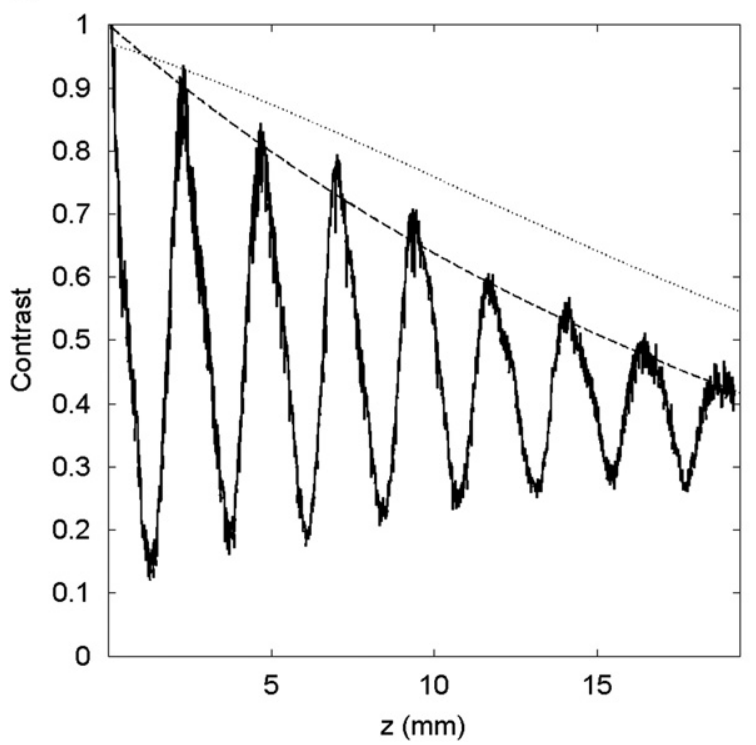

b

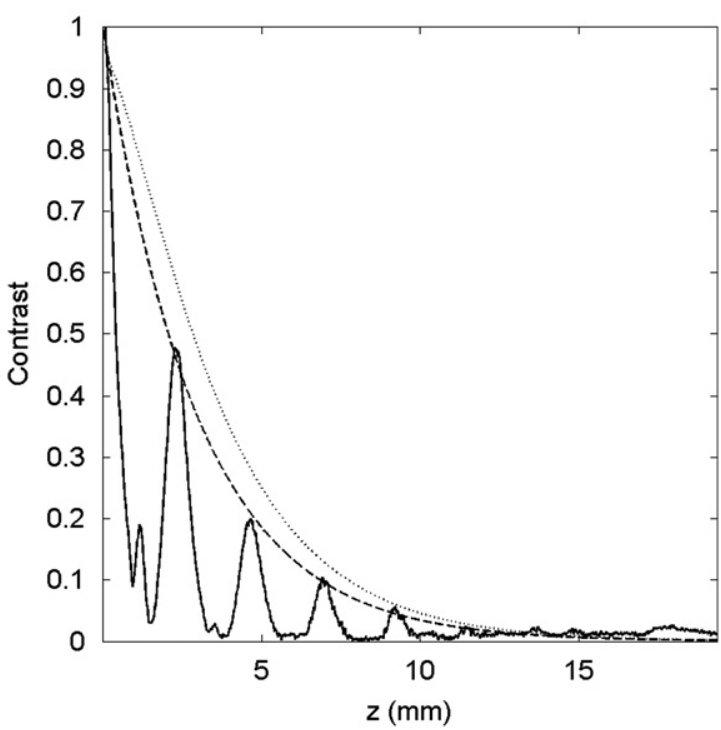

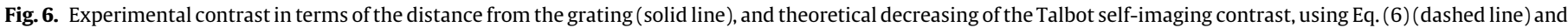
using Eq. (7) (dotted line), for (a) low level roughness steel; and (b) high level roughness steel.

shown in Table 1). As it can be observed, the contrast of the selfimages and the exponential decreasing ruled by the topographical measurements lead to very close results. As it was expectable, the fitting theory-experiment is more accurate for the high roughness steel tape grating, since the model was developed for high roughness limit. However, for the low roughness steel tape grating, the adjustment is also quite good. Therefore, we have demonstrated that controlling the topographical surface quality of the steel tapes where the grating is engraved, it is possible to improve its optical behavior.

\section{Conclusions}

Steel tape gratings are unavoidable used in some applications, such as optical encoders with long measuring distances. However, the contrast of Talbot self-images decreases strongly with the distance from the grating due to the inherent roughness on the steel surface. In previous works, the authors theoretically determined an equation for computing the contrast of the Talbot selfimages showing that it depends on the surface roughness. In this work, we have performed an experimental verification of this effect showing that steel can be a good optical material in diffractive optics for some applications where the use of chrome-over-glass gratings is not indicated. We have determined the roughness parameters of steel from direct topological measurements and we have compared the resulting theoretical decreasing with the experimental Talbot self-images contrast, showing that both results fit quite well.

\section{Acknowledgements}

The authors are grateful to Tomás Morlanes for his help. This work has been supported by the project DPI2008-02391 of the "Ministerio de Ciencia e Innovación" of Spain.

\section{References}

[1] Patorski K. Prog Opt 1989;27:1-108.

[2] Schirripa Spagnolo G, Ambrosini D, Paoletti D. J Opt A-Pure Appl Opt 2002;4: 376-80.

[3] Torcal-Milla FJ, Sanchez-Brea LM, Bernabeu E. Opt Commun 2008;281: 5647-52.

[4] Torcal-Milla FJ, Sanchez-Brea LM, Bernabeu E. Appl Opt 2007;46:3668-73.

[5] Sanchez-Brea LM, Torcal-Milla FJ, Bernabeu E. Opt Commun 2007;278:23-7.

[6] Talbot WHF. Philos Mag 1836;9:401-7.

[7] Beckmann P, Spizzichino A. The scattering of electromagnetic waves from rough surfaces. Artech House; 1987.

[8] Ogilvy A. Theory of wave scattering from random rough surfaces. IOP; 1991.

[9] Perez-Quitián F, Lutemberg A, Rebollo MA. Appl Opt 2006;45:4821-5.

[10] Salgado-Remacha FJ, Sanchez-Brea LM, Alvarez-Rios FJ, Bernabeu E. Appl Opt 2010;49:1750-6.

[11] Sanchez-Brea LM, Torcal-Milla FJ, Bernabeu E. Appl Opt 2007;46:5027-33. 